\title{
THE PUTT AND THE PENDULUM: Ironic Effects of the Mental Control of Action
}

\author{
Daniel M. Wegner, Matthew Ansfield, and Daniel Pilloff \\ University of Virginia
}

\begin{abstract}
People sometimes find themselves making movement errors that represent the ironic opposite of what they intended to do. These studies examined this tendency in the case of putting a golf ball and swinging a handheld pendulum, and found that ironic errors were particularly likely when participants who were instructed to avoid them tried to do so under mental load or physical load. The idea that such errors may be prompted by a monitoring process that increases sensitivity to the most undesirable outcome of an intention was supported by the finding of a tendency for ironic errors to be more evident when participants were allowed to monitor their action visually than when they could not.
\end{abstract}

My worst thoughts, then, were confirmed. —Edgar Allan Poe (1843), The Pit and the Pendulum

Many of our actions transpire as planned, but some fall to errorand an annoying few plummet all the way down past mere error to confirm our worst thoughts. This article is about actions in the final group, ones that unfold in precisely the most unwanted way. These are all too familiar: When we think, "Don't spill this" as we carry a full cup, for example, we may trigger the very spill we wish to avoid. When we near a precipice, in turn, and think not to fall, we often teeter toward the brink. And we are likewise vexed in sports when we find that the error we most want to overcome seems to happen recurrently. Our bodies mock us as we double dribble, slice into the rough, miss our first serve, and otherwise work our way out of the record books and into blooper videos.

The theory of ironic processes of mental control (Wegner, 1994) accounts for such errors by suggesting that both intentional and counterintentional effects arise from the same control system, which consists of two processes that usually work together to promote the intentional control of the mind: An intentional operating process searches for mental contents that will yield the desired state, and an ironic monitoring process searches for mental contents that signal the failure to achieve the desired state. Both processes increase the accessibility of the mental contents for which they are searching, and this increases the likelihood that they will instigate relevant thoughts, emotions, and actions. Whereas the operating process is effortful, consciously guided, and effective, however, the monitoring process is usually unconscious, less demanding of mental effort, and correspondingly less influential.

The two processes produce mental control by interacting over time. The operating process creates the desired change by filling the mind with thoughts and sensations that are relevant to the desired state. The monitoring process meanwhile searches surreptitiously for mental

Matthew Ansfield is now at Southern Methodist University.

Address correspondence to Daniel M. Wegner, Department of Psychology, Gilmer Hall, University of Virginia, Charlottesville, VA 22903; e-mail: dwegner@virginia.edu. contents relevant to failure because these indicate when control is needed. If the monitor finds indications of control failure, it reinitiates the operating process. Because the monitor stays watchful of lapses in control, however, it keeps the mind sensitive to the conditions that indicate that intentional mental control is failing. Thus, when mental capacity is undermined for any reason and the operating process is limited, the sensitivity supplied by the monitor can ironically create the mental state that corresponds to control failure. Under mental load, in other words, intentions to control the mind unleash a monitoring system that not only searches for the failure of mental control but then tends itself to create that failure.

Evidence for ironic effects has been observed in the mental control of thought (Wegner \& Erber, 1992), mood (Wegner, Erber, \& Zanakos, 1993), relaxation (Wegner, Broome, \& Blumberg, 1996), sleep (Ansfield, Wegner, \& Bowser, 1996), and memory (Macrae, Bodenhausen, Milne, \& Ford, 1997), as well as in other domains (Wegner, 1994, 1997). The present studies were designed to determine whether this explanation extends to ironies of action. It makes sense that the search processes posited by the theory might not be limited to cognitive and emotional effects, but could also have motor effects. As suggested by ideomotor action theories (Arnold, 1946; Carpenter, 1884; W. James, 1890 ), as well as by more contemporary theories of cognition in action (e.g., Bargh, Chen, \& Burrows, 1996; Dijksterhuis \& van Knippenburg, in press; Wilson \& Capitman, 1982), perhaps the accessibility of mental contents about an action yields readiness for action. If so, then both intentional operating processes and ironic monitoring processes could have motor effects-with the relatively weaker monitoring processes yielding observable (and erroneous) action whenever mental load undermines the operating processes that promote intentional action.

In the present studies, the venues chosen for testing this notion were the control of putting in golf and the control of a handheld pendulum. Problems in the control of putting are generally appreciated even by nongolfers, but the control of pendulum movement requires a bit more introduction. Actually, interest in the pendulum first centered on its ostensibly occult powers. Superstition holds that the swing pattern of a pendulum held over a pregnant woman's belly, for example, foretells the sex of the child, and there once were theories of how a pendulum would swing over ore samples to indicate their metallic content (Jastrow, 1937). Such magical properties of the pendulum were debunked by Chevreul (1833), who found that the pendulum swing typically follows the holder's attention and expectations. For the pendulum to "work," the holder has to be looking at it and suspending it from a hand or arm so that muscle movement can influence the swing. Still, even when people are aware of their possible influence, there is a sense in which the pendulum movement is unintentional. Just thinking about a circular or left-to-right swing, for example, seems to be sufficient for many people to generate that pattern (Easton \& Shor, 1975, 1976, 1977). Carpenter (1884) observed that when a person thinks about the hour of the day while swinging a pendulum inside a glass, it often strikes the hour even while the person has no sense of doing this on purpose. 
In a first study of ironic processes in pendulum movement (Wegner \& Ansfield, 1994, cited in Wegner, 1994), 8 male and 8 female participants each held a pendulum under instructions not to let it move in the direction of an axis drawn on a sheet of paper. Observers recorded the maximum distance that the point of the pendulum traveled along the forbidden axis. Also, half of the participants were given a mental load during the task, having been instructed to count down from 1,000 by $7 \mathrm{~s}$. Consistent with prediction, participants under load showed greater movement in the forbidden direction $(M=3.11 \mathrm{~cm})$ than those without load $(M=2.56 \mathrm{~cm}), t(17)=2.40, p=.03$. This pilot experiment lacked a comparison of unwanted movement with changes in movement in other directions. The present studies examined both unwanted and irrelevant movements under conditions of load-first for the putt, then for the pendulum.

\section{EXPERIMENT 1: THE PUTT}

Part of putting a golf ball is considering ways in which the putt could go wrong. The ironic process theory suggests that putting the ball in precisely such unwanted ways should be most likely to occur when the intentional operating process is disabled by mental load, and when the ironic monitoring process is enabled by attention to the ongoing action. This experiment focused on both variables, manipulating both load and visual monitoring. Evidence for the role of load in producing ironic effects was mentioned earlier, but it is worth noting that there is also some evidence for the role of visual monitoring. Chevreul (1833) observed, as did Easton and Shor (1975), that participants who look away from the pendulum no longer produce the involuntary movement. A similar finding was reported by R.J. James (1983) for the control of postural tremor. The present study examined, then, whether visual monitoring and mental load magnify overshooting a golf putt when this is specifically proscribed.

\section{Method}

\section{Participants and design}

Undergraduates (42 male and 41 female) participated as required for an introductory psychology course. Informed consent was obtained, and rights of participants were protected in this and the second study. Under the restriction that the proportion of males and females remain roughly the same across conditions, participants were randomly assigned to conditions of a 2 (mental load: load vs. no load) $\times 2$ (visual monitoring: present vs. absent) design.

\section{Procedure}

Each participant was given the opportunity to putt a golf ball on a carpeted surface in a room lit only by ultraviolet light. This lighting allowed the manipulation of visual monitoring, in that some participants used a putter that glowed orange under the light (monitoring present), whereas others used a black putter that was difficult to see (monitoring absent). The ball glowed yellow, and a target "glow spot" that was $4 \mathrm{~cm}$ in diameter and lying on the floor $2 \mathrm{~m}$ away glowed blue. To reduce visibility from glowing clothing, the participant and experimenter donned black sweatsuits. Each participant took warm-up putts with each putter under no special instruction.

Accuracy was recorded when the participant took a baseline putt with one of the two putters, under instructions to "land the ball on the glow spot." Either before or after the baseline, the participant took an experimental putt. For this putt, the participant used the same putter under instructions to "land the ball on the glow spot, but be particularly careful not to hit the ball past the glow spot; don't overshoot the glow spot." Participants in the mental-load condition in addition were asked to keep a six-digit number in mind and report it after the experimental putt.

\section{Results}

Overshooting was measured as the difference between the experimental and control putts in centimeters past the glow spot. (Withinsubjects analysis of the experimental and control putts yielded conclusions paralleling those of the difference score analysis, so this analysis is presented for brevity; no significant effects were observed for control putts in a separate analysis.) In an analysis of variance (ANOVA), a marginally significant sex difference was found, with males undershooting $(M=-11.67 \mathrm{~cm})$ and females overshooting $(M=19.61 \mathrm{~cm})$, $F(1,75)=3.64, p<.06, M S E=5,523.13$. Sex of participant was retained as a factor in the analysis, but did not significantly interact with any other factor; order of experimental and control trials was not retained, as it showed no significant main or interactive effects.

There was a main effect for mental load, $F(1,75)=4.14, p<.05$, with participants under mental load overshooting more $(M=20.79$ $\mathrm{cm})$ than those without load $(M=-11.43 \mathrm{~cm})$. This effect is consistent with the ironic process prediction, given that all subjects were asked not to overshoot. Also, although the interaction of load with visual monitoring was not significant, individual comparisons indicated that mental load tended to increase overshooting in the visual-monitoring condition, $F(1,75)=3.41, p<.07$, whereas it did not do so in the nomonitoring condition, $F<1$. With visual monitoring, there was marginally more overshooting under load $(M=32.87 \mathrm{~cm})$ than without load $(M=-9.07 \mathrm{~cm})$. Without visual monitoring, there was only a little more overshooting with load $(M=8.71 \mathrm{~cm})$ than without $(M=-13.78$ $\mathrm{cm})$. Although other sources of proprioception remained available to participants, the reduction of visual monitoring tended to reduce the role of load as a cause of ironic error.

An analysis performed to test absolute departure of putts from the midline revealed no significant main or interactive effects; neither load nor visual monitoring, then, influenced the degree to which shots strayed from a straight line. Finally, an analysis including participants' experience with golf as an independent variable in the design showed that the load effect on overshots was somewhat more pronounced for novices than for golfers, but not significantly so.

\section{EXPERIMENT 2: THE PENDULUM}

This study focused on the prediction that ironic effects of movement control would be enhanced under conditions of load, either mental or physical. Individuals were asked not to move a handheld pendulum in a particular direction or were asked to hold it steady without mention of a direction, and were given a load or not. For some participants, the load consisted of counting backward from 1,000 by $3 \mathrm{~s}-$ a mental load much like the loads employed in prior ironic process research (Wegner, 1994). For other participants, the load involved holding a brick in the outstretched opposite arm-a physical load like the loads one might encounter in work, sports, or other circumstances requiring precise movement during exertion. It was expected that a load of either kind would intensify the effect of ironic processes, and so increase the degree of unwanted movement. 


\section{Method}

\section{Participants and design}

Undergraduates (42 male and 42 female) were recruited as in the prior study. Under the restriction that the proportion of males and females stay similar across cells, participants were randomly assigned to the cells of a 2 (load: load vs. no load) $\times 2$ (instruction: prevent sideways movement vs. hold it steady) $\times 2$ (load type: mental vs. physical) design.

\section{Apparatus and procedure}

A video camera was aimed upward beneath a $36-\mathrm{cm}$-square glass plate, on which a grid was centered. The grid was a $12.5-\mathrm{cm}$ square, with $x$ and $y$ axes highlighted. Grid lines were $0.5 \mathrm{~cm}$ apart, and there was a 3-mm, green transparent dot at the intersection of the axes. The pendulum was a pointed crystal weighing $2 \mathrm{~g}$. It was attached to $50 \mathrm{~cm}$ of light nylon line. A knot was tied $30 \mathrm{~cm}$ from the crystal to mark where the pendulum was to be held. The participant clasped the line as though it were a tennis racket - in the palm of the dominant hand, with the pendulum end extending forward over the second knuckle of the index finger. The apparatus was calibrated for each participant's height, so that the glass plate rested $2 \mathrm{~cm}$ below the pendulum when the participant's forearm was parallel to the floor with elbow bent and not resting on the body.

For the prevent-sideways-movement condition, participants were instructed, "When I say to begin, you will be asked not to let the pendulum move by holding it as steady as you can over the green center point. Your task is not to let the pendulum move, keeping it as steady as possible. The pendulum should be held as steady as possible over the center spot, and you should not let it move in the direction paralleling the horizontal line on the page in front of you. You should try to hold it about an inch above the grid. The trial will last $30 \mathrm{~s}$. Again, do not move it sideways, in the direction paralleling the horizontal line." The experimenter pointed to identify the $x$-axis as the forbidden direction of movement.

Participants in the mental-load condition were told, "Also, you are to count backward in your head from 1,000 by $3 \mathrm{~s}$ (that is, 1,000, 997, 994 , and so on). At the end of the trial, I will ask you the last number you reached, so remember that number after I say stop." Those in the physical-load condition were asked to hold a common brick $(2.2 \mathrm{~kg})$ in the nonpendulum hand during the trials. The brick was held in the upturned hand with the forearm parallel to the floor and elbow bent and not resting against the body.

Participants in the steady-hold condition received parallel instructions, with the exception that they were asked merely to hold the pendulum as steady as possible-with no mention of a forbidden direction. Each participant completed five trials, with a 30 -s rest period following each trial.

\section{Movement measure}

An observer viewed the video of each trial and counted the number of perceptible movements of the pendulum parallel to each of the two axes. Diagonal or circular movements that could not be judged as going in one or the other direction were also counted. A second observer coded data for 10 of the participants, and the two observers reached an effective reliability of .75 . The proportion of movements on each axis was calculated as the average, across the five trials, of the ratio of the number of movements on that axis to the total number of movements $(x+y+$ others $)$.

\section{Results}

A $2 \times 2 \times 2 \times 2$ ANOVA assessed effects of load, instruction, load type, and repeated measures of direction ( $x$ vs. $y$ ) on the movement proportion. A significant main effect was observed for direction of movement, $F(1,76)=42.40, p<.001, M S E=.02$. More movement occurred on the $x$-axis $(M=.51)$ than on the $y$-axis $(M=.38)$ overall, perhaps because of a greater ease with which the arm is moved sideways as compared with to and fro in this position. Significant interactions were found for instruction by direction, $F(1,76)=10.25, p=$ .002 , and load by direction, $F(1,76)=6.43, p=.01$, but these effects were both qualified by the predicted interaction of load, instruction, and direction of movement, $F(1,76)=3.80, p=.05$ (see Fig. 1). Tests of simple main effects for $x$-axis movement revealed that more occurred when participants tried not to move on the $x$-axis under load $(M=.59)$ than without load $(M=.50), F(1,81)=6.26, p=.01, M S E=$ .01 , and that more such movement occurred when they tried not to move on the $x$-axis under load $(M=.59)$ than when they simply held steady under load $(M=.47), F(1,81)=13.68, p=.001$. In short, more unwanted movements occurred when participants attempted to avoid such movement under load.

The proportion of $y$-axis movement was reduced when participants tried to avoid $x$-axis movement under load, as would be expected given the negative correlation between the $x$-axis and $y$-axis movement proportions $(r=-.69)$. Less $y$-axis motion occurred for participants trying to avoid $x$-axis movement with load $(M=.32)$ than without load $(M=.40)$, $F(1,81)=8.50, p=.005$. The proportion of $y$-axis movement was also lower during avoidance of $x$-axis movement under load $(M=.32)$ than for participants holding steady under load $(M=.39), F(1,81)=6.50, p=$ .01 . The decrement in proportion of $y$-axis movements was due to the relative increment in $x$-axis movements, as a similar decrement was not observed in a separate analysis of raw number of $y$-axis movements.

No significant main effect or interactions were found for load type, so the observed dependency of ironic movement errors on load was general across both mental and physical load. It would be interesting to know whether the effects of physical load are limited to ironic action errors, or might also extend to ironic mental control of other kinds. Finally, the sex difference observed in the prior study was not present in Experiment 2, as there were no main or interactive effects for sex.

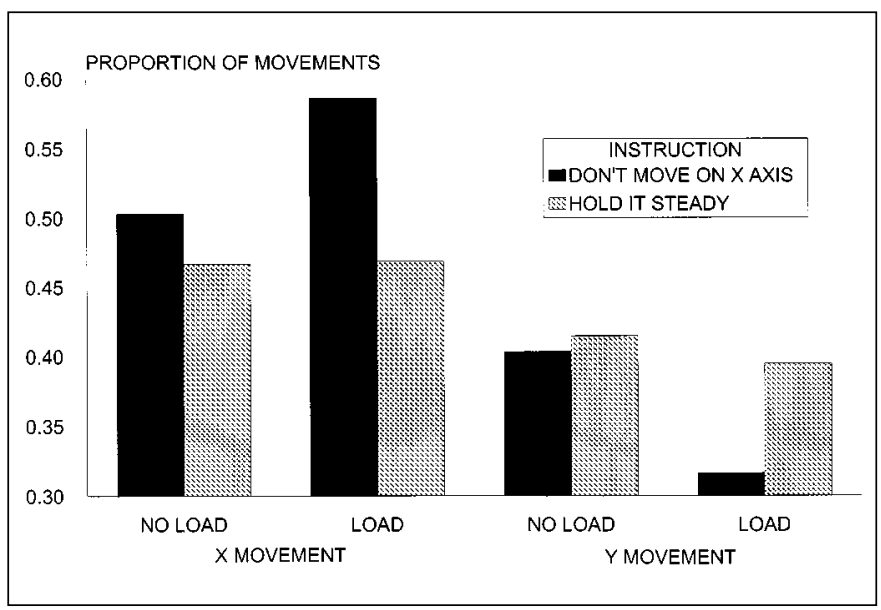

Fig. 1. Proportion of movements on the $x$-axis and $y$-axis as a function of load and instruction in Experiment 2. 


\section{GENERAL DISCUSSION}

Trying not to perform simple actions under load can prompt the occurrence of those actions. These studies showed that the attempt not to overshoot a golf putt can induce a significant overshot when mental load is present, and that the attempt not to swing a pendulum in a certain direction provokes just such a swing - significantly so in the presence of mental load or physical load. As the ironic process theory predicts (Wegner, 1994), it appears that distraction can undermine motor control to produce not only erroneous movement, but precisely the least wanted movement. Also consistent with the theory is the marginally significant indication in Experiment 1 that this ironic effect is more likely when people are specifically allowed visual access to monitor their behavior.

An alternative to the ironic process account of these effects might be suggested by the ideomotor action theory (e.g., Arnold, 1946; Easton \& Shor, 1975; Gordon \& Rosenbaum, 1984). It could be that when an erroneous movement is suggested to participants (by the admonition to avoid it), the action is primed and thus becomes more likely to occur. This account does not explain why mental or physical load would enhance the effect, however. Most conceivable paths from imagining action to performing action should require cognitive capacity, and they should therefore be impaired by the imposition of load, not enhanced by it. Indeed, Easton and Shor (1976) found that the effect of simply imagining directional movement on pendulum oscillation was undermined by distraction. The present findings indicate that load has the paradoxical effect of increasing ironic errors, specifically when those errors are being avoided as a result of instruction, and a simple priming account falls short of explaining this phenomenon (cf. Ansfield \& Wegner, 1996).

The errors of the putt and the pendulum, in sum, resemble all too well many blunders of everyday life. The ironic process approach to these things reminds us, though, that even when we are at our most clumsy-fumbling, lurching, wobbling, tripping, flailing, and otherwise expressing the dregs of our motor repertoires-there is one redeeming possibility: We do these things because we know better and have simply made the mistake of trying not to err under load.

Acknowledgments-This research was supported by Grant MH 49127 from the National Institute of Mental Health. We thank Hillary Gelfman, Jennifer George, and Elisa Wilson for their help. A preliminary description of a subset of the Experiment 2 data appeared in Ansfield and Wegner (1996).

\section{REFERENCES}

Ansfield, M.E., \& Wegner, D.M. (1996). The feeling of doing. In P.M. Gollwitzer \& J.A. Bargh (Eds.), The psychology of action: Linking cognition and motivation to behavior (pp. 482-506). New York: Guilford Press.

Ansfield, M.E., Wegner, D.M., \& Bowser, R. (1996). Ironic effects of sleep urgency. Behaviour Research and Therapy, 34, 523-531.

Arnold, M.B. (1946). On the mechanism of suggestion and hypnosis. Journal of Abnormal and Social Psychology, 41, 107-128.

Bargh, J.A., Chen, M., \& Burrows, L. (1996). Automaticity of social behavior: Direct effects of trait construct and stereotype activation on action. Journal of Personality and Social Psychology, 71, 230-244.

Carpenter, W.G. (1884). Principles of mental physiology, with their applications to the training and discipline of the mind and study of its morbid conditions. New York: Appleton.

Chevreul, M.E. (1833). Lettre à M. Ampère sure une classe particulaires. Review des Deux Mondes, 2, 258-266.

Dijksterhuis, A., \& van Knippenburg, A. (in press). Automatic social behavior or how to win a game of Trivial Pursuit. Journal of Personality and Social Psychology.

Easton, R.D., \& Shor, R.E. (1975). Information processing analysis of the Chevreul pendulum illusion. Journal of Experimental Psychology: Human Perception and Performance, 1, 231-236.

Easton, R.D., \& Shor, R.E. (1976). An experimental analysis of the Chevreul pendulum illusion. Journal of General Psychology, 95, 111-125.

Easton, R.D., \& Shor, R.E. (1977). Augmented and delayed feedback in the Chevreul pendulum illusion. Journal of General Psychology, 97, 167-177.

Gordon, A.M., \& Rosenbaum, D.A. (1984). Conscious and subconscious arm movements: Application of signal detection theory to motor control. Bulletin of the Psychonomic Society, 22, 214-216.

James, R.J. (1983). Acute effects of instrumental augmented feedback on self-control and automaticity of postural tremor associated with local muscle fatigue. Behavioral Engineering, 8, 138-145.

James, W. (1890). Principles of psychology. New York: Holt.

Jastrow, J. (1937). Chevreul as psychologist. Scientific Monthly, 44, 487-496.

Macrae, C.N., Bodenhausen, G.V., Milne, A.B., \& Ford, R.L. (1997). On the regulation of recollection: The intentional forgetting of stereotypical memories. Journal of Personality and Social Psychology, 72, 709-719.

Wegner, D.M. (1994). Ironic processes of mental control. Psychological Review, 101, 34 52.

Wegner, D.M. (1997). When the antidote is the poison: Ironic mental control processes. Psychological Science, 8, 148-150.

Wegner, D.M., Broome, A., \& Blumberg, S. (1997). Ironic effects of trying to relax under stress. Behaviour Research and Therapy, 35, 11-21.

Wegner, D.M., \& Erber, R. (1992). The hyperaccessibility of suppressed thoughts. Journal of Personality and Social Psychology, 63, 903-912.

Wegner, D.M., Erber, R., \& Zanakos, S. (1993). Ironic processes in the mental control of mood and mood-related thought. Journal of Personality and Social Psychology, 65, 1093-1104.

Wilson, T.D., \& Capitman, J.A. (1982). Effects of script availability on social behavior Personality and Social Psychology Bulletin, 8, 11-20.

(RECEIVED 6/11/96; REVISION ACCEPTED 12/9/97) 